\title{
THE SPECTRAL EQUALITY FOR UPPER TRIANGULAR OPERATOR MATRICES WITH UNBOUNDED ENTRIES
}

\author{
Deyu Wu, Alatancang Chen and Tin-Yau Tam
}

Abstract. Let

$$
M_{C}=\left[\begin{array}{ll}
A & C \\
0 & B
\end{array}\right]: D\left(M_{C}\right) \subset X \times X \rightarrow X \times X
$$

be a $2 \times 2$ unbounded upper triangular operator matrix on the complex Hilbert space $X \times X$. We investigate the conditions under which $\sigma\left(M_{C}\right)=\sigma(A) \cup \sigma(B)$ holds in the diagonally dominant $\left(D\left(M_{C}\right)=D(A) \times D(B)\right)$ and upper dominant case $\left(D\left(M_{C}\right)=D(A) \times D(C)\right)$. Some necessary and sufficient conditions are obtained. The results generalize some results of Han, Du, and Barraa in the bounded case.

Mathematics subject classification (2010): 47B47, 47A10.

Keywords and phrases: Spectral equality, upper triangular operator matrices, null space.

\section{REFERENCES}

[1] M. BARRAA AND M. BOUMAZGOUR, A note on the spectrum of an upper triangular operator matrix, Proc. Amer. Math. Soc. 131 (2003), 3083-3088.

[2] H. K. Du And J. PAN, Perturbation of spectrums of $2 \times 2$ operator matrices, Proc. Amer. Math. Soc. 121 (1994), 761-776.

[3] P. R. Halmos, A Hilbert space problem book, Springer-Verlag, New York, 1982.

[4] J. K. HAN, H. Y. LEE AND W. Y. LEE, Invertible completions of $2 \times 2$ upper triangular operator matrices, Proc. Amer. Math. Soc. 129 (2000), 119-123.

[5] V. HARDT AND R. MENNICKEN, On the spectrum of unbounded off-diagonal $2 \times 2$ operator matrices in Banach spaces, recent advances in operator theory (Groningen, 1998), 243-266, Oper. Theory Adv. Appl. 124, Birkhuser, Basel, 2001.

[6] V. Hardt, A. Konstantinov and R. Mennicken, On the spectrum of the product of closed operators, Math. Nachr. 215 (2000), 91-102.

[7] I. S. HWANG AND W. Y. LEE, The boundedness below of $2 \times 2$ upper triangular operator matrices, Integral Equations Operator Theory 39 (2001), 267-276.

[8] J. J. Huang, A. Chen AND H. WAng, The symplectic eigenfunction expansion theorem and its application to the plate bending equation, Chinese Physics B, 16 (2009), 3616-3623.

[9] T. Kato, Perturbation Theory for Linear Operators, Springer-Verlag: Berlin Heidelberg, 1995.

[10] R. NAGEL, Towards a "matrix theory" for unbounded operator matrices, Math. Z. 201 (1989), 57-68.

[11] L. SAKHNOVICH, Effective construction of a class of positive operators in Hilbert space, which do not admit triangular factorization, J. Funct. Anal. 263 (2012), 803-817.

[12] C. TRETTER, Spectral Theory of Block Operator Matrices and Applications, Imperial College Press, London, 2008.

[13] C. TRETTER, Spectral inclusion for unbounded block operator matrices, J. Funct. Anal. 256 (2009), 3806-3829.

[14] D. Y. WU AND A. CHEN, Invertibility of nonnegative Hamiltonian operator with unbounded entries, J. Math. Anal. Appl. 373 (2011), 410-413.

[15] D. Y. WU AND A. CHEN, Spectral inclusion properties of the numerical range in a space with an indefinite metric, Linear Algebra Appl. 435 (2011), 1131-1136. 